\title{
Declines in Prevalence of Human Papillomavirus Vaccine-Type Infection Among Females after Introduction of Vaccine — United States, 2003-2018
}

\author{
Hannah G. Rosenblum, MD ${ }^{1,2}$; Rayleen M. Lewis, $\mathrm{MPH}^{2,3}$; Julia W. Gargano, $\mathrm{PhD}^{2}$; Troy D. Querec, $\mathrm{PhD}^{4}$;
} Elizabeth R. Unger, $\mathrm{PhD}, \mathrm{MD}^{4}$; Lauri E. Markowitz, $\mathrm{MD}^{2}$

Human papillomavirus (HPV) is the most common sexually transmitted infection in the United States (1). Although most infections resolve without clinical sequalae, persistent HPV infection can cause cervical, other anogenital, and oropharyngeal cancers and anogenital warts. HPV vaccination has been recommended in the United States at age 11-12 years since 2006 for females and since 2011 for males. Catch-up vaccination is recommended through age 26 years.* A quadrivalent vaccine ( $4 \mathrm{vHPV})$ targeting types $6,11,16$, and 18 was mainly used until 2015, when a 9-valent vaccine (9vHPV), targeting the same four types as $4 \mathrm{vHPV}$ and five additional types (31, 33, 45, 52, and 58), was introduced; 9vHPV has been the only vaccine available in the United States since the end of 2016 (2). HPV vaccination coverage has increased but remains lower than that of other vaccinations recommended for adolescents (3). A decrease in prevalence of $4 \mathrm{vHPV}$ types detected in cervicovaginal swabs among young females from the prevaccine era (2003-2006) to 2007-2010 in the National Health and Nutrition Examination Survey (NHANES) was an early indicator of vaccine impact (2) and was also observed in later periods $(4,5)$. NHANES data from 2017-2018 were included in this analysis to update HPV prevalence estimates among females aged 14-34 years. From the prevaccine era to 2015-2018, significant decreases in 4vHPV-type prevalence occurred among females aged $14-19$ years (88\%) and 20-24 years (81\%). In sexually experienced females, $4 \mathrm{vHPV-type} \mathrm{prevalence}$ decreased in those who reported receiving $\geq 1 \mathrm{HPV}$ vaccine dose (97\% among those aged $14-19$ years, $86 \%$ among those aged $20-24$ years) and in those who reported no vaccination (87\% among those aged $14-19$ years, $65 \%$ among those aged 20-24 years). Significant declines among unvaccinated females suggest herd effects. These data show increasing impact of HPV vaccination in the United States. HPV vaccination is a critical prevention tool against HPV infection, anogenital warts, and HPV-attributable precancers and cancers. HPV vaccination is highly effective and is recommended routinely at age 11-12 years and through 26 years for persons not already vaccinated.

NHANES is an ongoing cross-sectional survey conducted by CDC's National Center for Health Statistics designed to monitor

\footnotetext{
${ }^{*}$ https://www.cdc.gov/vaccines/hcp/acip-recs/vacc-specific/hpv.html\#recs
}

the health and nutrition of the U.S. non-institutionalized civilian population. Data collection for NHANES was approved by the National Center for Health Statistics Research Ethics Review Board. This activity was reviewed by CDC and was conducted consistent with applicable federal law and CDC policy. ${ }^{\dagger}$ Demographic and HPV vaccination information are obtained during in-home interviews. Sexual behavior information is obtained via audio computer-assisted self-interview, and self-collected cervicovaginal specimens are obtained in mobile examination centers. ${ }^{\Im}$ CDC determines HPV DNA genotypes from these specimens using L1 consensus polymerase chain reaction testing followed by type-specific hybridization to detect $37 \mathrm{HPV}$ types and $\beta$-globin ( 6 ). Samples negative for both HPV and $\beta$-globin are considered inadequate and were excluded from analysis. Data from 2003 through 2018 were analyzed in 4-year periods: 2003-2006, 2007-2010, 2011-2014, and 2015-2018. Age groups were categorized as 14-19, 20-24, 25-29, and 30-34 years. Self-reported race/ethnicity was categorized as non-Hispanic White, non-Hispanic Black, Mexican American, ${ }^{* *}$ or other races. Self- or parent-reported vaccination history was analyzed as ever vaccinated (receipt of $\geq 1$ dose). Sexual behaviors analyzed included ever having had sex ${ }^{\dagger \dagger}$ (sexually experienced) and number of lifetime sex partners (fewer than three or three or more partners ${ }^{\$ \mathbb{S}}$ ). HPV prevalences $\$ \mathbf{9}$ and $95 \%$ confidence intervals (CIs) were estimated for each 4-year period for the following type categories: $4 \mathrm{vHPV}$ types, ${ }^{* * *}$ five additional 9vHPV

$\dagger 45$ C.F.R. part 46.102(l)(2), 21 C.F.R. part 56; 42 U.S.C. Sect. 241(d); 5 U.S.C. Sect. 552a; 44 U.S.C. Sect. 3501 et seq.

$\$$ https://wwwn.cdc.gov/nchs/data/nhanes/2017-2018/manuals/2017_MEC_ Interviewers_Procedures.pdf

9 Research Use Only Linear Array HPV Genotyping Test which tests for HPV $6,11,16,18,26,31,33,35,39,40,42,45,51,52[\mathrm{XR}], 53,54,55,56,58$, $59,61,62,64,66,67,68,69,70,71,72,73,81,82,83,84,89$, and IS39.

** NHANES oversamples subpopulations to increase reliability and precision of estimates for specific racial/ethnic groups. Because of changes in oversampling methodology of persons who are Hispanic across the study period, the National Center for Health Statistics recommends that researchers do not report estimates for other Hispanic persons (i.e., Hispanic persons who are not Mexican American persons) using data before 2007 or for Hispanic subgroups other than Mexican American, in any survey cycle through 2018. Therefore, only estimates for persons who are Mexican American could be presented for this entire study period.

†† Anal, oral, or vaginal sex.

$\$ \$$ Includes same sex and opposite sex partners.

95 Prevalence estimates with a relative standard error of $>30 \%$ were noted and considered unstable.

*** HPV 6, 11, 16, and 18. 
types, ${ }^{\dagger \dagger}$ non-4vHPV types, ${ }^{\$ \$ \$}$ and non-9vHPV types. 999 Prevalence ratios (PRs) and adjusted prevalence ratios (aPRs), comparing 2015-2018 with 2003-2006, were estimated using logistic regression. For females aged 14-19 years, estimates were adjusted a priori for race/ethnicity and ever having had sex. For females aged 20-24, 25-29, and 30-34 years, estimates were adjusted a priori for race/ethnicity and number of lifetime sex partners. Distributions of participant characteristics and behaviors were estimated for each 4-year period; 2015-2018 was compared with 2003-2006 using Wald F tests. Among sexually experienced females, $4 \mathrm{vHPV}$-type prevalence in each 4-year period was estimated overall, by age group and vaccination history. Complex survey analytic methods were used to account for the survey design; analyses used examination weights.**** Statistical significance was defined as $\mathrm{p}<0.05$, or $95 \%$ CIs that did not include one for PRs. Prevalence estimates with relative standard error $>30 \%$ were noted for stability concerns. Data management and analysis were performed using SAS (version 9.4; SAS Institute) and SUDAAN (version 11.0; RTI International). Because the analyses required restricted-use data, the data were accessed through CDC's Research Data Center. ${ }^{+\dagger \dagger}$

Prevalence of $4 \mathrm{vHPV}$-type infection decreased $88 \%$ among females aged $14-19$ years, from $11.5 \%$ during 2003-2006 (prevaccine era) to $1.1 \%$ during 2015-2018 $(\mathrm{aPR}=0.12 ; 95 \% \mathrm{CI}=0.06-0.26)$ (Table 1). Prevalence of five additional 9vHPV types in this age group decreased 65\%, from $8.4 \%$ during 2003-2006 to 2.3\% during 2015-2018 $(\mathrm{aPR}=0.35 ; 95 \% \mathrm{CI}=0.18-0.65)$. Among females aged 20-24 years, 4vHPV-type prevalence decreased $81 \%$, from $18.5 \%$ during $2003-2006$ to $3.3 \%$ during $2015-2018$ $(\mathrm{aPR}=0.19 ; 95 \% \mathrm{CI}=0.09-0.40)$; the prevalence of the five additional 9vHPV types did not decline significantly. Among females in older age groups, no statistically significant differences in the prevalence of $4 \mathrm{vHPV}$ or the five additional 9vHPV types were noted from 2003-2006 to 2015-2018. Regarding HPV types not targeted by vaccine, among females aged 14-19 years, the prevalence of non-4vHPV types decreased from $31.2 \%$ during the prevaccine era to $20.9 \%$ during 2015-2018 ( $\mathrm{aPR}=0.72 ; 95 \% \mathrm{CI}=0.57-0.92)$; the prevalence of non-9vHPV types decreased from $29.0 \%$ to $20.6 \%(\mathrm{aPR}=0.77 ; 95 \% \mathrm{CI}=0.61-0.98)$. The prevalences of non- $4 \mathrm{vHPV}$ and non-9vHPV types in the other age groups

$\dagger \dagger$ HPV 31, 33, 45, 52, and 58.

$\$ ₫ \$$ Thirty-three HPV types detected using linear array that are not HPV 6, 11,16 , or 18 .

999 Twenty-eight types detected using linear array that are not HPV 6, 11, 16, $18,31,33,45,52$, or 58 .

**** https://wwwn.cdc.gov/nchs/data/nhanes/analyticguidelines/11-16-analyticguidelines.pdf

t†t† https://www.cdc.gov/rdc/index.htm were not statistically significantly different during 2015-2018 compared with 2003-2006.

Reported receipt of $\geq 1 \mathrm{HPV}$ vaccine dose increased from 2007-2010 to 2015-2018 in all age groups (Table 2). The percentages of females aged 14-19 years reporting ever having had sex and three or more lifetime partners were significantly lower during 2015-2018 than during 2003-2006. Among females in older age groups, during 2003-2006 and 2015-2018, >90\% reported ever having had sex; the percentage with three or more lifetime partners did not change significantly. The distribution of race/ethnicity was different in most age groups during 2015-2018 than during the prevaccine era.

Among sexually experienced females, the prevalence of $4 \mathrm{vHPV}$-type infections among those aged 14-19 years decreased from $19.3 \%$ during 2003-2006 to the following during 2015-2018: overall, $1.5 \%(\mathrm{PR}=0.08$; 95\% CI $=0.03-0.22)$; those vaccinated, $0.6 \%(\mathrm{PR}=0.03 ; 95 \% \mathrm{CI}=0.00-0.25)$; those unvaccinated, $2.4 \%(\mathrm{PR}=0.13 ; 95 \% \mathrm{CI}=0.03-0.48)$ (i.e., a $97 \%$ decrease among those vaccinated and an $87 \%$ decrease among those unvaccinated). In sexually experienced females aged 20-24 years, 4vHPV-type prevalence decreased from $17.9 \%$ during 2003-2006 to $2.5 \%$ $(\mathrm{PR}=0.14 ; 95 \% \mathrm{CI}=0.05-0.41)$ among those vaccinated and to $6.3 \%(\mathrm{PR}=0.35 ; 95 \% \mathrm{CI}=0.14-0.85)$ among those unvaccinated during 2015-2018 (i.e. an 86\% decrease among those vaccinated and a $65 \%$ decrease among those unvaccinated). Smaller, nonsignificant decreases in 4vHPV-type prevalences were observed among vaccinated females in older age groups: from $12.4 \%$ to $4.6 \%$ among those aged $25-29$ years and from $9.0 \%$ to $4.4 \%$ among those aged $30-34$ years (Figure) (Supplementary Table, https://stacks.cdc.gov/view/ cdc/104147).

\section{Discussion}

During 2015-2018, the prevalence of 4vHPV types was 88\% lower than that during the prevaccine era among females aged $14-19$ years and $81 \%$ lower among those aged $20-24$ years after adjustment for sexual behavior and race/ethnicity. The decline among females aged 14-19 years was first observed within 4 years of vaccine introduction (2), and prevalence has continued to decline in subsequent years (4). The decline among females aged 20-24 years was first observed within 6 years of vaccine introduction (6); 10 years after introduction, through 2016, 4vHPV-type prevalence among women aged 20-24 years had decreased $71 \%$ (5). This report, through 12 years after vaccine introduction, shows sustained low 4vHPV-type prevalence among females aged 14-19 years and larger declines among those aged 20-24 years.

Very few participants surveyed during 2017-2018 would have received 9vHPV; therefore, it is likely too early for 
Morbidity and Mortality Weekly Report

TABLE 1. Prevalence of human papillomavirus (HPV) infection among females aged 14-34 years, by age group and survey years — National Health and Nutrition Examination Survey, United States, 2003-2018*

\begin{tabular}{|c|c|c|c|c|c|c|}
\hline \multirow{2}{*}{$\begin{array}{l}\text { Age group (yrs) and } \\
\text { HPV types }\end{array}$} & $\begin{array}{l}\text { Prevaccine era } \\
2003-2006\end{array}$ & 2007-2010 & 2011-2014 & 2015-2018 & \multicolumn{2}{|c|}{ Comparison of $2015-2018$ with $2003-2006$} \\
\hline & \multicolumn{4}{|c|}{$\%(95 \% \mathrm{Cl})$} & PR $(95 \% \mathrm{Cl})$ & $\operatorname{aPR}(95 \% \mathrm{CI})^{\dagger}$ \\
\hline 14-19 & $\mathrm{n}=1,363$ & $n=740$ & $\mathrm{n}=797$ & $n=666$ & $0.10(0.05-0.21)$ & $0.12(0.06-0.26)$ \\
\hline $4 \mathrm{vHPV} \S$ & $11.5(9.1-14.4)$ & $5.0(3.8-6.6)$ & $3.3(1.9-5.8)$ & $1.1(0.5-2.4)^{9}$ & & \\
\hline $\begin{array}{l}\text { Additional five types in } \\
9 \mathrm{vHPV}^{* *}\end{array}$ & $8.4(6.6-10.6)$ & $6.1(4.4-8.5)$ & $5.3(3.4-8.4)$ & $2.3(1.3-4.1)$ & $0.28(0.15-0.51)$ & $0.35(0.18-0.65)$ \\
\hline Non-4vHPV ${ }^{+\dagger}$ & $31.2(27.9-34.8)$ & $25.3(21.4-29.5)$ & $25.5(21.3-30.2)$ & $20.9(16.9-25.6)$ & $0.67(0.53-0.84)$ & $0.72(0.57-0.92)$ \\
\hline Non-9vHPV§§ & $29.0(26.0-32.2)$ & $24.4(20.8-28.4)$ & $24.7(20.6-29.4)$ & $20.6(16.6-25.3)$ & $0.71(0.57-0.90)$ & $0.77(0.61-0.98)$ \\
\hline $\begin{array}{l}20-24 \\
4 \mathrm{vHPV}\end{array}$ & $\begin{array}{r}\mathrm{n}=432 \\
18.5(14.9-22.8)\end{array}$ & $\begin{array}{r}\mathrm{n}=445 \\
19.9(15.4-25.3)\end{array}$ & $\begin{array}{r}n=442 \\
7.2(4.7-11.1)\end{array}$ & $\begin{array}{c}n=368 \\
3.3(1.7-6.3)^{9}\end{array}$ & $0.18(0.09-0.35)$ & $0.19(0.09-0.40)$ \\
\hline $\begin{array}{l}\text { Additional five types in } \\
\text { 9vHPV** }\end{array}$ & $16.5(11.3-23.4)$ & $13.8(10.2-18.2)$ & $13.2(8.8-19.4)$ & $10.2(7.2-14.4)$ & $0.62(0.38-1.02)$ & $0.62(0.38-1.01)$ \\
\hline Non-4vHPV ${ }^{\dagger \dagger}$ & $50.7(43.4-58.0)$ & $57.4(51.3-63.3)$ & $55.8(49.9-61.6)$ & $49.9(42.3-57.5)$ & $0.98(0.80-1.21)$ & $0.97(0.80-1.18)$ \\
\hline Non-9vHPV§§ & $47.6(40.7-54.6)$ & $54.9(48.9-60.8)$ & $53.4(47.8-58.8)$ & $47.1(39.7-54.7)$ & $0.99(0.80-1.22)$ & $0.97(0.79-1.18)$ \\
\hline $25-29$ & $n=403$ & $n=414$ & $\mathrm{n}=395$ & $n=430$ & $0.77(0.46-1.29)$ & $0.85(0.50-1.46)$ \\
\hline $4 \mathrm{vHPV} \S$ & $11.8(8.8-15.6)$ & $13.1(10.0-17.2)$ & $8.8(6.3-12.1)$ & $9.1(5.8-14.0)$ & & \\
\hline $\begin{array}{l}\text { Additional five types in } \\
9 \mathrm{vHPV}^{* *}\end{array}$ & $10.8(7.3-15.7)$ & $13.1(9.7-17.3)$ & $13.9(10.5-18.1)$ & $11.6(8.1-16.3)$ & $1.07(0.64-1.79)$ & $0.99(0.58-1.67)$ \\
\hline Non-4vHPV ${ }^{\dagger \dagger}$ & $43.8(38.9-48.9)$ & $48.6(43.7-53.6)$ & $43.7(37.7-49.9)$ & $45.2(39.2-51.4)$ & $1.03(0.87-1.23)$ & $1.05(0.86-1.27)$ \\
\hline Non-9vHPV§§ & $39.8(34.8-45.0)$ & $44.7(40.0-49.4)$ & $42.0(36.2-48.0)$ & $42.1(36.6-47.9)$ & $1.06(0.88-1.27)$ & $1.07(0.88-1.31)$ \\
\hline $30-34$ & $\mathrm{n}=389$ & $\mathrm{n}=433$ & $\mathrm{n}=433$ & $\mathrm{n}=413$ & $0.65(0.38-1.11)$ & $0.67(0.37-1.21)$ \\
\hline $4 \mathrm{vHPV} \S$ & $9.5(6.7-13.2)$ & $8.9(6.5-11.9)$ & $7.1(5.1-9.9)$ & $6.2(4.0-9.5)$ & & \\
\hline $\begin{array}{l}\text { Additional five types in } \\
9 \mathrm{vHPV}^{* *}\end{array}$ & $9.8(7.1-13.5)$ & $6.8(4.7-9.9)$ & $6.9(4.6-10.0)$ & $6.9(4.4-10.8)$ & $0.70(0.41-1.21)$ & $0.68(0.37-1.27)$ \\
\hline Non-4vHPVt† & $44.5(39.1-50.1)$ & $37.8(31.6-44.5)$ & $39.2(33.6-45.0)$ & $34.7(29.1-40.8)$ & $0.78(0.63-0.96)$ & $0.82(0.67-1.00)$ \\
\hline Non-9vHPV§§ & $40.4(35.0-46.0)$ & $36.1(30.3-42.3)$ & $38.2(32.7-44.0)$ & $31.9(26.6-37.6)$ & $0.79(0.64-0.98)$ & $0.83(0.67-1.03)$ \\
\hline
\end{tabular}

Abbreviations: aPR = adjusted prevalence ratio; $\mathrm{Cl}=$ confidence interval; $4 \mathrm{vHPV}=$ quadrivalent $\mathrm{HPV}$ vaccine; 9vHPV $=9$-valent $\mathrm{HPV}$ vaccine; $\mathrm{PR}=$ prevalence ratio.

* All analyses were weighted using the National Health and Nutrition Examination Survey examination sample weights.

${ }^{\dagger}$ Adjustments for aPR: females aged 14-19 years, race/ethnicity and ever had sex; females aged 20-24, 25-29, and 30-34 years, race/ethnicity and number of lifetime sex partners (fewer than three or three or more).

$\S$ HPV 6, 11, 16, or 18.

I Relative standard error $>30 \%$ and $\leq 50 \%$, considered unstable.

* HPV 31, 33, 45, 52, or 58

t+ Thirty-three HPV types detected using linear array that are not HPV 6, 11, 16, or 18.

$\S \S$ Twenty-eight HPV types detected using linear array that are not HPV $6,11,16,18,31,33,45,52$, or 58.

9vHPV vaccination to explain all of the decline in prevalence of the additional 9vHPV-type infections among females aged 14-19 years. The significant declines also in prevalences of non-4vHPV-type and non-9vHPV-type infections in this age group from the prevaccine era to 2015-2018 suggest lower exposure to HPV; this is consistent with the decrease in reported sexual behaviors from 2003-2006 to 2015-2018 in the current report. Lower exposure might have contributed to some of the observed decrease in 4vHPV-type prevalence among females aged 14-19 years. Among females aged 20-24 years, an age group with no significant changes in reported sexual behavior, a dramatic decline in 4vHPV-type prevalence from the prevaccine era to 2015-2018 occurred without significant change in non-4vHPV-type or non-9vHPV-type prevalence, demonstrating vaccine impact in the absence of potential changes in HPV exposure.

In addition to significantly lower 4vHPV-type prevalence among sexually experienced vaccinated females compared with those in the prevaccine era, $4 \mathrm{vHPV}$-type prevalence was also lower among unvaccinated females: $87 \%$ in females aged $14-19$ years and $65 \%$ in those aged $20-24$ years. These findings suggest strong herd effects, or indirect protection of unvaccinated females, as reported in previous NHANES analyses and in data from other countries $(4,7)$. The herd effects are likely attributable to less circulation of vaccine-type HPV because of both female and male vaccination in the United States $(2,3)$.

The findings in this report are subject to at least three limitations. First, differences in sexual behavior were noted among females aged 14-19 years in 2015-2018 compared with 2003-2006. To account for this, prevalence ratios were adjusted for sexual behaviors, and a subanalysis was restricted to sexually experienced females; however, residual confounding might be present. Second, self- and parent-reported vaccination history could have resulted in misclassification, which might bias findings, including those related to herd effects (8). Finally, small sample size resulted in limited precision for certain subgroup analyses. 
TABLE 2. Characteristics of females aged 14-34 years, by age group and survey years - National Health and Nutrition Examination Survey, United States, 2003-2018*

\begin{tabular}{|c|c|c|c|c|}
\hline \multirow[b]{2}{*}{ Age group (yrs) and characteristic } & \multicolumn{4}{|c|}{$\%(95 \% \mathrm{Cl})$} \\
\hline & 2003-2006 & 2007-2010 & 2011-2014 & 2015-2018 \\
\hline \multicolumn{5}{|l|}{$14-19$} \\
\hline HPV vaccine history: $\geq 1$ dose $^{\dagger}$ & $\mathrm{N} / \mathrm{A}$ & $34.1(28.4-40.3)$ & $54.7(49.6-59.7)$ & $54.3(49.2-59.4)$ \\
\hline Ever had sex ${ }^{\S}$ & $54.0(50.9-57.0)$ & $50.3(45.0-55.6)$ & $48.2(43.0-53.3)$ & $45.4(41.0-49.8)$ \\
\hline Three or more lifetime partners $§$ & $25.6(22.5-29.0)$ & $22.6(19.9-25.5)$ & $23.4(19.4-27.9)$ & $17.5(14.3-21.2)$ \\
\hline \multicolumn{5}{|l|}{ Race/Ethnicity ${ }^{\S}$} \\
\hline White, non-Hispanic & $65.5(58.9-71.6)$ & $60.1(54.4-65.6)$ & $57.7(50.2-64.9)$ & $51.6(43.3-59.8)$ \\
\hline Black, non-Hispanic & $14.8(11.0-19.7)$ & $15.3(12.1-19.0)$ & $14.5(10.2-20.3)$ & $14.6(10.7-19.4)$ \\
\hline Mexican American & $10.1(7.6-13.5)$ & $12.4(8.8-17.2)$ & $13.6(10.6-17.5)$ & $15.1(11.1-20.2)$ \\
\hline Other races & $9.5(6.8-13.1)$ & $12.2(9.3-15.9)$ & $14.1(11.3-17.5)$ & $18.7(15.0-23.1)$ \\
\hline \multicolumn{5}{|l|}{$20-24$} \\
\hline HPV vaccine history: $\geq 1$ dose $^{\dagger}$ & $\mathrm{N} / \mathrm{A}$ & $17.8(12.4-24.9)$ & $43.0(36.0-50.4)$ & $59.9(53.0-66.5)$ \\
\hline Ever had sex & $91.4(86.1-94.8)$ & $91.9(88.3-94.5)$ & $91.4(86.9-94.5)$ & $94.9(90.4-97.3)$ \\
\hline Three or more lifetime partners & $60.7(53.7-67.2)$ & $71.8(66.1-77.0)$ & $68.4(63.9-72.5)$ & $67.5(62.3-72.4)$ \\
\hline \multicolumn{5}{|l|}{ Race/Ethnicity } \\
\hline White, non-Hispanic & $61.6(54.6-68.2)$ & $56.7(49.0-64.2)$ & $55.7(46.9-64.1)$ & $55.3(47.5-62.7)$ \\
\hline Black, non-Hispanic & $15.7(11.1-21.9)$ & $15.9(12.3-20.5)$ & $16.6(11.4-23.6)$ & $13.1(9.1-18.6)$ \\
\hline Mexican American & $11.5(8.2-15.9)$ & $12.9(9.0-18.0)$ & $9.6(6.6-13.8)$ & $13.0(8.2-20.1)$ \\
\hline Other races & $11.2(7.7-15.9)$ & $14.5(10.3-19.9)$ & $18.1(13.8-23.4)$ & $18.6(14.2-23.9)$ \\
\hline \multicolumn{5}{|l|}{$25-29$} \\
\hline HPV vaccine history: $\geq 1$ dose $^{\dagger}$ & N/A & $7.8(5.5-11.1)$ & $24.8(19.7-30.7)$ & $40.7(34.6-47.1)$ \\
\hline Ever had sex & $95.0(91.7-97.1)$ & $95.6(92.0-97.7)$ & $95.4(92.5-97.2)$ & $94.1(89.8-96.7)$ \\
\hline Three or more lifetime partners & $73.2(66.4-79.0)$ & $71.9(66.7-76.6)$ & $71.8(64.9-77.8)$ & $72.1(65.7-77.6)$ \\
\hline \multicolumn{5}{|l|}{ Race/Ethnicity ${ }^{\S}$} \\
\hline White, non-Hispanic & $65.0(57.9-71.6)$ & $63.6(56.0-70.5)$ & $56.4(47.8-64.7)$ & $53.8(48.0-59.5)$ \\
\hline Black, non-Hispanic & $12.5(8.9-17.4)$ & $12.3(8.7-17.2)$ & $11.9(8.6-16.2)$ & $15.7(11.5-21.1)$ \\
\hline Mexican American & $12.1(8.6-16.7)$ & $10.0(7.2-13.6)$ & $11.5(7.6-16.9)$ & $10.9(6.9-16.7)$ \\
\hline Other races & $10.4(7.1-14.9)$ & $14.2(10.4-19.0)$ & $20.2(15.9-25.4)$ & $19.7(15.3-25.0)$ \\
\hline \multicolumn{5}{|l|}{$30-34$} \\
\hline HPV vaccine history: $\geq 1$ dose $^{\dagger}$ & $\mathrm{N} / \mathrm{A}$ & $3.7(2.0-6.7)$ & $7.0(4.6-10.4)$ & $18.9(13.9-25.2)$ \\
\hline Ever had sex & $98.4(95.4-99.4)$ & $97.4(93.0-99.1)$ & 99.1 (97.8-99.7) & $95.5(91.7-97.7)$ \\
\hline Three or more lifetime partners & $73.6(68.2-78.4)$ & $69.3(63.5-74.6)$ & $75.9(70.0-81.0)$ & $72.3(66.2-77.6)$ \\
\hline \multicolumn{5}{|l|}{ Race/Ethnicity ${ }^{\S}$} \\
\hline White, non-Hispanic & $61.8(55.6-67.7)$ & $58.1(48.5-67.0)$ & $59.7(53.1-66.0)$ & $53.4(44.8-61.8)$ \\
\hline Black, non-Hispanic & $15.8(11.6-21.2)$ & $14.4(9.9-20.5)$ & $13.6(10.2-18.0)$ & $12.7(9.2-17.1)$ \\
\hline Mexican American & $11.9(9.4-15.1)$ & $14.0(9.8-19.7)$ & $11.4(7.4-17.4)$ & $9.7(6.5-14.4)$ \\
\hline Other races & $10.4(6.8-15.7)$ & $13.5(9.3-19.2)$ & $15.2(12.6-18.2)$ & $24.3(18.5-31.2)$ \\
\hline
\end{tabular}

Abbreviations: $\mathrm{Cl}=$ confidence interval; $\mathrm{N} / \mathrm{A}=$ not applicable.

* All analyses were weighted using the National Health and Nutrition Examination Survey examination sample weights.

† Significant difference from 2015-2018 to 2007-2010 ( $p<0.05$ ).

$\S$ Significant difference from 2015-2018 to 2003-2006 ( $p<0.05)$.

This report adds to the robust data on the impact of the national HPV vaccination program, including herd effects. In addition to decreases in the prevalence of vaccine types, decreasing rates of cervical precancers and anogenital warts also have been demonstrated in the United States and other countries after introduction of HPV vaccination $(7,9)$. The COVID-19 pandemic has the potential to reverse gains made in HPV vaccination coverage in the United States, as indicated by lower adolescent vaccine orders in $2020(3,10)$. Efforts are needed to increase HPV vaccination to maintain the substantial progress of the vaccination program. Continued monitoring in NHANES will provide information to evaluate changes in U.S. vaccination recommendations and 9vHPV vaccine introduction on HPV prevalence as well as any setbacks attributable to the COVID-19 pandemic. HPV vaccination is a critical prevention tool against HPV infection, anogenital warts, and HPV-attributable precancers and cancers. HPV vaccination is highly effective and is recommended routinely at age 11-12 years and through age 26 years for persons not already vaccinated.

\section{Acknowledgments}

Juanita M. Onyekwuluje, Sonya Patel, Krystle L. Love, Division of High-Consequence Pathogens and Pathology, National Center for Emerging and Zoonotic Infectious Diseases, CDC; Carolyn Neal, National Center for Health Statistics, CDC.

Corresponding author: Hannah G. Rosenblum, HRosenblum@cdc.gov. 
FIGURE. Quadrivalent vaccine-type (4vHPV-type) prevalence among sexually experienced females aged 14-34 years, by age group, vaccination history, ${ }^{*}$ and survey years - National Health and Nutrition Examination Survey, United States, 2003-2018
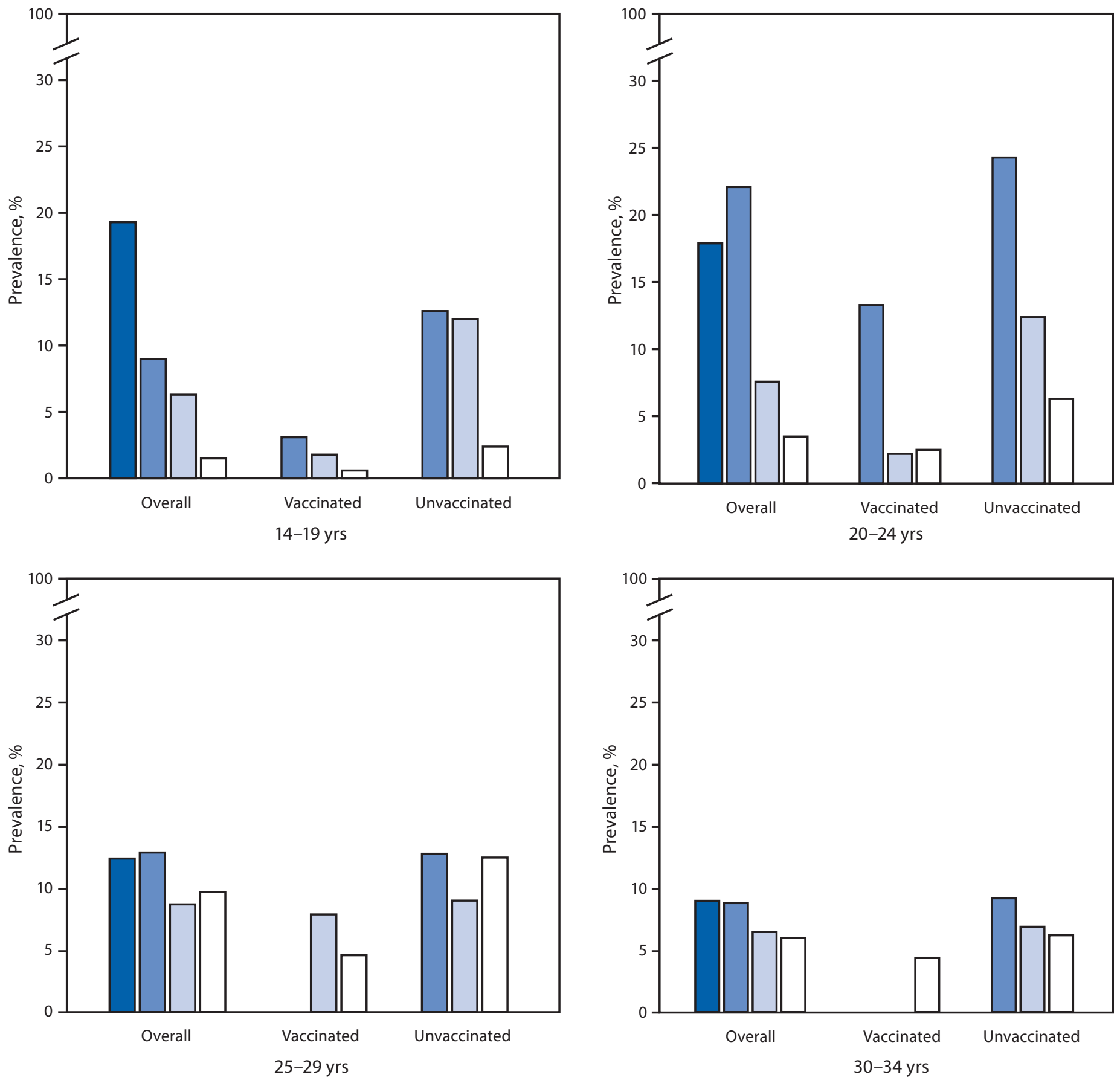

Prevaccine era: 2003-2006

$2007-2010$

$\square 2011-2014$

2015-2018

* Reported receipt of $\geq 1 \mathrm{HPV}$ vaccine dose.

† All analyses were weighted using the examination sample weights.

$\S$ Estimates are not shown for vaccinated females aged 25-29 years in 2007-2010 and for vaccinated females aged 30-34 years in 2007-2010 and 2011-2014 because of the low proportion of females in those age groups who were vaccinated. 


\begin{abstract}
${ }^{1}$ Epidemic Intelligence Service, CDC, ${ }^{2}$ Division of Viral Diseases, National Center for Immunization and Respiratory Diseases, CDC, ${ }^{3}$ Synergy America, Inc., Duluth, Georgia; ${ }^{4}$ Division of High-Consequence Pathogens and Pathology, National Center for Emerging and Zoonotic Infectious Diseases, CDC.
\end{abstract}

All authors have completed and submitted the International Committee of Medical Journal Editors form for disclosure of potential conflicts of interest. No potential conflicts of interest were disclosed.

\section{References}

1. Kreisel KM, Spicknall IH, Gargano JW, et al. Sexually transmitted infections among US women and men: prevalence and incidence estimates, 2018. Sex Transm Dis 2021. Epub January 23, 2021. PMID:33492089 https://doi.org/10.1097/OLQ.0000000000001355

2. Markowitz LE, Gee J, Chesson H, Stokley S. Ten years of human papillomavirus vaccination in the United States. Acad Pediatr 2018;18:S3-10. PMID:29502635 https://doi.org/10.1016/j.acap.2017.09.014

3. Elam-Evans LD, Yankey D, Singleton JA, et al. National, regional, state, and selected local area vaccination coverage among adolescents aged 13-17 yearsUnited States, 2019. MMWR Morb Mortal Wkly Rep 2020;69:1109-16. PMID:32817598 https://doi.org/10.15585/mmwr.mm6933a1

4. Oliver SE, Unger ER, Lewis R, et al. Prevalence of human papillomavirus among females after vaccine introduction-National Health and Nutrition Examination Survey, United States, 2003-2014. J Infect Dis 2017;216:594-603. PMID:28931217 https://doi.org/10.1093/infdis/jix244

5. McClung NM, Lewis RM, Gargano JW, Querec T, Unger ER, Markowitz LE. Declines in vaccine-type human papillomavirus prevalence in females across racial/ ethnic groups: data from a national survey. J Adolesc Health 2019;65:715-22. PMID:31515134 https://doi.org/10.1016/j.jadohealth.2019.07.003

6. Markowitz LE, Liu G, Hariri S, Steinau M, Dunne EF, Unger ER. Prevalence of HPV after introduction of the vaccination program in the United States. Pediatrics 2016;137:e20151968. PMID:26908697 https:// doi.org/10.1542/peds.2015-1968

7. Drolet M, Bénard É, Pérez N, et al.; HPV Vaccination Impact Study Group. Population-level impact and herd effects following the introduction of human papillomavirus vaccination programmes: updated systematic review and meta-analysis. Lancet 2019;394:497-509. PMID:31255301 https://doi.org/10.1016/S0140-6736(19)30298-3

\section{Summary}

What is already known about this topic?

Through 2016, human papillomavirus (HPV) vaccine-type prevalence decreased among young females after introduction of HPV vaccination in 2006.

What is added by this report?

Nationally representative data through 2018 indicate that HPV vaccine-type prevalence continues to decline among females aged $14-19$ (88\%) and 20-24 (81\%) years compared with before vaccination. The findings also show evidence of indirect protection of unvaccinated females through herd effects in these age groups.

What are the implications for public health practice?

HPV vaccination is a critical prevention tool against HPV infection, anogenital warts, and HPV-attributable precancers and cancers. HPV vaccination is highly effective and is recommended routinely at age $11-12$ years and through age 26 years for persons not already vaccinated.

8. Lewis RM, Markowitz LE. Human papillomavirus vaccination coverage among females and males, National Health and Nutrition Examination Survey, United States, 2007-2016. Vaccine 2018;36:2567-73. PMID:29650386 https://doi.org/10.1016/j.vaccine.2018.03.083

9. Gargano JW, Park IU, Griffin MR, et al.; HPV-IMPACT Working Group. Trends in high-grade cervical lesions and cervical cancer screening in 5 states, 2008-2015. Clin Infect Dis 2019;68:1282-91. PMID:30137283 https://doi.org/10.1093/cid/ciy707

10. Santoli JM, Lindley MC, DeSilva MB, et al. Effects of the COVID-19 pandemic on routine pediatric vaccine ordering and administrationUnited States, 2020. MMWR Morb Mortal Wkly Rep 2020;69:591-3. PMID:32407298 https://doi.org/10.15585/mmwr.mm6919e2 